Results $100 \%$ of the FY participants who responded to the post-project evaluation survey $(n=4)$ either agreed, or strongly agreed, with the following:

- I now have a greater understanding of how to complete a QIP.

- The amount of supervision I received was appropriate.

- I would recommend this model of learning to a colleague.

Each QIP demonstrated a positive impact on patient care, and included all of the core elements necessary to meet QI curriculum requirements.

Conclusion This work demonstrates how a blended approach can drive the development of QI skills to the point of expected competency, while also delivering successful QIPs that result in improved quality of patient care.

\section{USING FRAILTY REVIEWS TO MANAGE THE COVID-19 PANDEMIC IN AN EVIDENCE-BASED AND PERSON- CENTRED MANNER ACROSS BUCKINGHAMSHIRE CARE HOMES}

${ }^{1}$ Unoma Okoli* ${ }^{1}$ Shahina Juma ${ }^{1}$ Sheena Patel, ${ }^{1}$ Monice Hussain ${ }^{2}$ Ana Phelps, ${ }^{2}$ Beatrix Nagyova, ${ }^{2}$ Theresa Frang, ${ }^{2}$ Chi Yuen Yau, ${ }^{2}$ Veena Borkar, ${ }^{2}$ Stephanie Tanner, ${ }^{2}$ Jo Birrell. ${ }^{1}$ Buckinghamshire Clinical Commissioning Group, UK; ${ }^{2}$ Buckinghamshire Healthcare NHS Trust, UK

\subsection{6/leader-2021-FMLM.76}

Aim NICE COVID-19 guidelines NG191 recognise that frailer patients, including those with a higher Rockwood Clinical Frailty Scale ${ }^{1}$ (CFS), are seldom improved by hospital admission.

The project used a collaborative approach to undertake frailty reviews, aiming to reduce inappropriate hospital admissions. The reviews included discussing residents' preferred place of care and options in the event of deterioration, a Treatment Escalation Plan (TEP).

Method A collaborative was formed between care home managers, pharmacists and geriatricians to review frailty factors in residents over 65 years, excluding those with Learning Disabilities. Care homes with high risk of COVID-19 were prioritised. A Standard Operational Procedure was approved within one month. Using existing resources only, the project was delivered over 3 months. Communication was sent to GP practices, care homes and residents/families and consent was obtained. Templates were developed and education delivered.

Results Review outcomes were recorded electronically onto the GP clinical system. Care homes kept a copy and informed residents/families of the outcome. 595 residents were reviewed in 21 care homes.

Preliminary analysis for 71 residents reviewed May 2020 to April 2021 show:

- 42 survivors, 33 with severe frailty (CFS 7-9), 9 not severely frail $(\mathrm{CFS}<7)$. Out of total 28 residents with TEP indicating avoidance of hospital admission, 23 were not admitted and 5 had single hospital admission. 14 residents TEP included hospital management of whom 7 were admitted.

- 26 of the 29 deceased residents were severely frail. 96\% died in their preferred place of death.

Conclusion Clinical leadership demonstrated courage and capacity to challenge the status quo, improve organisational processes and innovate practice. This helped staff morale at a difficult time of immense pressure and positively enhanced care homes' profile and residents' experience.

\section{REFERENCE}

1. K Rockwood, et al. CMAJ 2005;173:489-495.

\section{USING PDSA (PLAN - DO - STUDY - ACT) MODEL TO INCREASE MEDICINE RECONCILIATION IN A TERTIARY CARE HOSPITAL OF A DEVELOPING COUNTRY}

Amber Sabeen, Abdul Aziz, Ambreen Amirali. Aga Khan University Hospital, Karachi Pakistan

\subsection{6/leader-2021-FMLM.77}

Objective Using PDSA (Plan - Do - Study - Act) model to increase medicine reconciliation in a tertiary care hospital of a developing country.

Background The WHO defines the medication reconciliation process as the formal process in which health care professionals partner with patients to ensure precise and complete medication information transfer at different interfaces of care'. Medication errors at time of a patient's hospital admission are common occurrence in health care settings. Majority of the error occurs during prescribing, dispensing or administration of medicines. In order to avoid these errors, medication reconciliation processes and systems have been employed by health care organizations throughout the world. Implementing medical reconciliation has successfully reduced medication error in many institutes.

Study Design and Methodology It was a quality improvement project conducted in the Department of Medicine, Aga Khan University Hospital Karachi. We included residents and interns working in Medicine department. The written proforma was distributed in three sessions for three consecutive weeks. The PDSA model was implemented for four months from February 2019 to May 2019 in the department of internal medicine.

Result The Medicine reconciliation compliance improved from 4\% in February 2019 to $96 \%$ in May 2019.

Conclusion With the help of PDSA cycle we advised and managed to implement quality improvement interventions and changes that resulted in significant improvement in medication reconciliation compliance. This strategy of PDSA cycle can be applied in other quality indicator projects also for increasing patient safety and decrease preventable harm. This project also shows that engaging the health care workers will overcome the resistance to change and implement sustainable systems.

\section{WHAT KIND OF PERCEPTIONS DO THE MEDICAL AND INFORMATION DIRECTORS HAVE ABOUT THE FACTORS INFLUENCING ON KNOWLEDGE MANAGEMENT IN HOSPITAL?}

Milla Kajanne. Researcher, University of Helsinki, Programme in Social and Health Research and Management, Finland

\subsection{6/leader-2021-FMLM.78}

Aims The aim of the study is to explore, how the systematic knowledge-based management and the assessment of effectiveness have been implemented in hospitals in Finland. What are the potential bottlenecks and practical challenges on the way 